\title{
Risky economic choices and frontal EEG asymmetry in the context of Reinforcer-Sensitivity-Theory-5
}

\author{
M. Rollwage ${ }^{1,2,3}$ (iD $\cdot$ H. Comtesse ${ }^{1,4} \cdot$ G. Stemmler ${ }^{1}$
}

Published online: 26 June 2017

(C) Psychonomic Society, Inc. 2017

\begin{abstract}
This study investigated individual risky choice behavior in a gambling task and its relation with traits proposed by the Reinforcer-Sensitivity-Theory-5 (RST-5; Corr \& McNaughton in Neuroscience and Biobehavioral Reviews, 36(10), 2339-2354, 2012) as well as with frontal EEG asymmetry. As assumed by the RST-5, the results showed independent influences of approach/avoidance and gain/loss sensitivities on participants' behavior in risky choices. Individual approach/avoidance sensitivity was predicted by trait measures of the behavioral approach system (BAS) and the behavioral inhibition system (BIS), while no such correlation was present for gain/loss sensitivity. EEG recordings revealed relatively stronger left-frontal cortical activity for trials with approach motivation compared to conflict and avoidance motivation. On the individual level, relatively stronger left-frontal cortical activity was associated with trait BAS. In addition, activity changes in frontal EEG asymmetry were associated relatively higher behavioral approach sensitivity. We conclude that frontal EEG asymmetry is an especially useful neuronal marker of BAS sensitivity and that the traits proposed by the
\end{abstract}

M. Rollwage

max.rollwage.16@ucl.ac.uk

1 Differential Psychology and Psychological Diagnostic, University of Marburg, Gutenbergstraße 18, 35032 Marburg, Germany

2 Wellcome Trust Centre for Neuroimaging, University College London, London WC1N 3BG, UK

3 Max Planck University College London Centre for Computational Psychiatry and Ageing Research, London WC1B 5EH, UK

4 Clinical and Biological Psychology, Catholic University Eichstaett-Ingolstadt, Eichstaett, Germany
RST-5 (measured by frontal EEG asymmetry and self-report) can be used to explain individual differences in risky choice behavior.

Keywords Reinforcement sensitivity $\cdot$ Behavioral approach system $\cdot$ Behavioral inhibition system $\cdot$ Loss $\cdot$ Valuation . Frontal EEG Asymmetry · Risk · Gambling task · Individual differences

Every day, people are faced with decisions about unknown outcomes, often including risks (e.g., when deciding where to invest money). Neuroeconomics achieved great insights in mechanisms that guide behavior in those situations and shed light onto the neuronal mechanisms underlying such behavioral outcomes (Glimcher \& Rustichini, 2004). In risky choices (with known probability, but unknown outcome), it is well established that people tend to weigh losses stronger than gains, resulting in risk-averse behavior (Novemsky \& Kahneman, 2005). However, less is known about individual differences of risky behavior. Since the valuation of appetitive and aversive stimuli and the motivation to approach appetitive and to avoid aversive outcomes are of major importance for such decisions, these concepts should be considered for explaining individual differences in risky choices (Ferguson, Heckman, \& Corr, 2011). The RST-5 (Corr \& McNaughton, 2012) fulfills this request, as it proposes sensitivity differences of neuronal systems, which mediate valuation (gain/loss) and motivational (approach/avoid) processes, and offers a neuronal explanation for personality differences.

There are several approaches to assess sensitivities of the assumed systems (Smillie, 2008). First, behavioral experiments can be used to trigger those systems and register the behavioral responses. Second, individual differences of traits, which are assumed to be associated with the systems, can be 
assessed (e.g., with questionnaires). Finally, neurobiological recordings can be used as indicators for the activity of these systems. In this study, we aim to combine all three approaches by assessing traits and neuronal markers (electroencephalography: EEG) which are assumed to be associated with the neuronal systems proposed by the RST- 5 , and to investigate how individual differences in those relate to differences in risky decision making.

\section{Assumptions of the RST-5}

According to the RST-5, two processing steps are necessary for producing motivated behavioral output: first valuation of appetitive and aversive stimuli, and then elicitation of the motivation to approach or avoid these stimuli. These processes are not identical, because the link between both depends on the contingency between actions and outcomes. For instance, a situation that contains the opportunity of producing rewards will elicit approach motivation, while the possibility that an expected reward is omitted will elicit avoidance behavior. Therefore, approach behavior results from reward and omission of punishment and avoidance behavior results from punishment and omission of reward. ${ }^{1}$ Thus, different brain-behavioral systems are assumed to control the processes of valuation and motivation, and therefore, the sensitivity for these processes should independently vary between individuals. So far, it is not clear how crucial this distinction is and to which extent valuation and motivational sensitivities are independent in terms of causation and personality description (Corr \& McNaughton, 2012).

The valuation of appetitive and aversive stimuli refers to the process of attaching an internal value to an external stimulus, and it was extensively investigated by using money (gains and losses) as reward and punishment (Glimcher \& Rustichini, 2004). Different valuation functions for gains and losses were assumed (Kahneman \& Tversky, 1979), whereas, on average, people seem to have a higher sensitivity to losses than to gains, resulting in risk-averse behavior (Corr \& McNaughton, 2012). Besides this general tendency, the RST-5 claims that there should be individual differences in the valuation of gains and losses, which has been reported in a study by Gonzalez and Wu (1999).

After transformation of the external stimulus into an internal value, approach or avoidance behavior will be elicited depending on the contingency between the action and the outcome (Corr \& McNaughton, 2012). The RST-5 postulates three basic brain-behavioral systems mediating approach and avoidance behavior. The BAS is conceptualized as the system

\footnotetext{
${ }^{1}$ To avoid linguistic confusion, we will refer to stimuli/events that elicit approach behavior (reward and omission of punishment) as attractors and to those that elicit avoidance behavior (punishment and omission of gain) as repulsors (Corr \& McNaughton, 2012).
}

responding to attractors and mediating approach behavior (Corr, 2008). The fight-flight-freeze system (FFFS) should mediate reactions to repulsors and evokes active avoidance/ escape behavior (Corr, 2008). The BIS is assumed to respond to goal conflicts, leading to inhibition of the prepotent behaviors, promoting risk assessment and passive avoidance (Corr, 2008). Moreover, BIS activation is believed to selectively potentiate avoidance tendencies, while, in a simple approachavoidance conflict, BIS activation will favor avoidance over approach, and thus lead to a bias towards risk-averse behavior (Corr \& McNaughton, 2008).

Hence, FFFS and BIS are both involved in mediating avoidance behavior. However, it has been emphasized that avoidance should be rarely mediated solely by the FFFS since this system is assumed to control responses to threat when avoidance is the only motivation present (Corr, DeYoung, \& McNaughton, 2013). Most situations in modern societies include appetitive and aversive incentives at the same time (Wacker, Heldmann, \& Stemmler, 2003), for example, when deciding whether to ask ones' boss for a promotion and hoping for agreement or to hold the tongue, fearing rejection. Hence, avoidance in day-to-day behavior might be better explained in terms of passive avoidance, mediated by the BIS (DeYoung, 2010).

Variation in the sensitivity of BAS, FFFS, and BIS should result in differences in the tendency to actively approach, actively avoid, or passively avoid stimuli/events. Importantly, these motivational sensitivities are conceptually distinct from the valuation sensitivities for gains and losses. Nevertheless, a concrete behavioral output will always be determined by the concurrence of different systems and sensitivities (see Fig. 1 in Hall, Chong, McNaughton, \& Corr, 2011), as for producing observable behavior both stages need to interact (Corr \& McNaughton, 2012). Thus, experimental designs need to be thoroughly planned to disentangle both stages of sensitivity.

\section{Behavioral measurement of brain-behavioral systems proposed by the RST-5}

In order to assess those sensitivities separately and to control for reciprocal influences, Hall et al. (2011) designed a twophase gambling task, in which participants were presented with risky choices with a 50:50 chance of winning or losing money. Participants needed to decide if to accept or to reject the gamble. The two phases of the experiment solely differed in the contingency between actions and the consequence concerning the gamble. In Phase 1, participants needed to move a mouse cursor to a target and click on it to accept the gamble. In the second phase, participants needed to do the same active response to reject gambles. Hall et al. proposed that each response can be interpreted as approach behavior, motivated in Phase 1 by production of gains and in Phase 2 by prevention of losses. No response would always be interpreted 
as avoidance behavior that would be motivated by losses in Phase 1 and, in Phase 2, by the omission of gains.

In this way an orthogonalization of gain and loss valuation and the elicited motivational tendency was achieved, making it possible to assess approach versus avoidance (approach/ avoidance) sensitivity independently of gain versus loss (gain/loss) sensitivity, and vice versa. It is important to note that within this paradigm the avoidance behavior is a passive form of avoidance and therefore most probably driven by the BIS (see discussion above). This paradigm was incorporated for the present study in order to measure gain, loss, approach, and avoidance sensitivity on the behavioral level.

\section{Neuronal correlates of motivational and valuation sensitivities}

In addition to the behavioral manifestation of valuation and motivational sensitivities, it was suggested to probe their neurobiological correlates (e.g., Smillie, 2008). Especially the neuronal correlates of approach and avoidance motivation were extensively researched. For instance, the motivational direction model (Davidson, 1995) assumes that left- and right-frontal brain regions are part of two separate neuronal systems underlying approach and withdrawal motivation. If so, the comparison between activity at homologous left- and right-frontal regions (frontal EEG asymmetry) could be used to indicate the relative activity of these motivational systems. ${ }^{2}$ Moreover, individual differences in frontal EEG asymmetry were proposed as a trait-like measure of affective reactivity (Davidson, 1995).

The motivational systems conceptualized by Davidson show strong overlap with those proposed by the RST-5, although they are not identical (Coan \& Allen, 2003; HarmonJones \& Allen, 1997; Wacker et al., 2003). There are at least two competing models (De Pascalis, 2008), the behavioral activation behavioral inhibition model of anterior asymmetry (BBMAA, Wacker et al., 2003) and the bilateral frontal BAS model (Harmon-Jones \& Allen, 1997) that both try to allocate the brain-behavioral systems of the RST-5 to left- and rightfrontal cortical regions.

The BBMAA proposes a general behavioral activation system, which is located in left-frontal cortical areas and mediates approach behavior (BAS activity) but also pure withdrawal or active avoidance behavior of the FFFS (Wacker, Chavanon, Leue, \& Stemmler, 2008; Wacker et al., 2003). The authors argue that pure FFFS activity is rarely observed and therefore left-frontal activity should be more strongly linked to the BAS

\footnotetext{
${ }^{2}$ In most studies, the EEG alpha power $(8-12 \mathrm{~Hz}$ ) was used as indicator for cortical deactivation/inverted indicator of activity (Knyazev, 2007; Oakes et al., 2004). For better readability, we will use the term cortical activity as a synonym for reduced alpha power.
}

(Wacker et al., 2003). Behavioral inhibition and resolution of goal conflicts (BIS activity) were specifically linked to rightfrontal cortical areas. In addition to studies that associated leftfrontal activation with approach motivation (Davidson, Ekman, Saron, Senulis, \& Friesen, 1990; Sobotka, Davidson, \& Senulis, 1992), Wacker et al. (2003; Wacker et al., 2008) showed that pure withdrawal/active avoidance motivation was associated with relatively stronger leftfrontal cortical activity, but experienced conflict, with relatively right-frontal activity. Moreover, conflict in monetary choice tasks resulted in increased right-frontal cortical activity (Neo \& McNaughton, 2011). Further supporting the BBMAA, individual differences in self-report measures of BAS and BIS sensitivity were associated with individual differences in relative left- and right-frontal cortical activity, respectively (Shackman, McMenamin, Maxwell, Greischar, \& Davidson, 2009; Sutton \& Davidson, 1997).

In contrast, the bilateral frontal BAS model (Harmon-Jones \& Allen, 1997) links bilateral frontal cortical activity to the BAS and proposes that not only Davidson's approach system but also the withdrawal system is related to the BAS, as an approach to safety should also be BAS-mediated. This idea was based on the finding that individuals with greater bilateral frontal cortical activity showed higher psychometric BAS scores (Harmon-Jones \& Allen, 1997; Hewig, Hagemann, Seifert, Naumann, \& Bartussek, 2004, 2006).

Whether gain and loss sensitivities could also be assessed by specific EEG signals remains an open question. Studies showed evidence for separate systems of gain and loss valuation (Trepel, Fox, \& Poldrack, 2005). The ventral striatum and the amygdala were linked to valuation of gains and losses, respectively (Breiter, Aron, Kahneman, Dale, \& Shizgal, 2001; Knutson \& Peterson, 2005; Yacubian et al., 2006). In addition, the ventromedial prefrontal cortex (vmPFC) appears to code for both, appetitive and aversive stimuli, and for the overall value of behavioral options (Monosov \& Hikosaka, 2012; Plassmann, O’Doherty, \& Rangel, 2010; Tom, Fox, Trepel, \& Poldrack, 2007). However, it seems unlikely that the frontal EEG activity would be strongly affected by those areas (Davidson, 2004).

\section{The current study}

The current study used the gambling paradigm presented by Hall et al. (2011) in order to measure independent behavioral manifestations of gain/loss and approach/avoidance sensitivity. This behavioral paradigm was complemented by selfreport measures of BAS, BIS, and FFFS sensitivity to investigate if the individual choice behavior could be explained by these personality traits. Moreover, the correlation of behaviorally measured gain/loss and approach/avoidance sensitivities with self-report measures could give insights if the separation 
of the valuation and the motivational processing stages is valid or whether both sensitivity stages collapse in terms of personality description.

For a comparison of the different models of frontal cortical activity, $^{3}$ the EEG was recorded at the time of gamble presentation, during which the motivational systems should have been triggered. Arguably, the recorded EEG signal should reflect the activity of these systems. The models make different predictions about the expected pattern of frontal cortical activity, according to the elicited motivational tendency. Formulated as an open research question, we also explored whether neuronal correlates of gain/loss valuation could be found.

Finally, we were interested if frontal cortical activity could be used to predict individual behavior in risky choices, as already presented by Gianotti et al. (2009). Previous studies investigated frontal EEG asymmetry in connection to risk taking per se, without differentiating between valuation and motivation processes. We examined to which extent individual sensitivities to gains/losses and approach/avoidance would be predicted by frontal EEG asymmetry.

\section{Method}

\section{Participants}

The final sample comprised $N=50$ nonsmoking, right-handed female undergraduate psychology students (mean age $=23.3$ years, range: 18-40) without self-reported psychiatric disorder. Eight subjects were excluded from the original sample $(N$ $=58)$ because they did not complete the whole experiment $(N$ $=2)$, there were technical problems with the EEG recording $(N$ $=1$ ), or they doubted that they would be paid according to their earnings in the gambling task $(N=5)$. Additionally to the fulfillment of course requirements, participants were paid according to their gains in the gambling task. Average payment was $M=7.00 €$ (range: 4.76-10.64 $€$ ). The study was approved by the local ethics committee. All volunteers gave written informed consent.

\section{Experimental design and gambling task}

In a within-subjects design, participants were presented gambles with a 50:50 chance of winning or losing money and

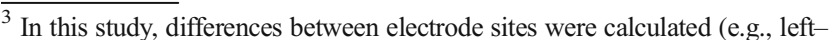
right frontal cortical activity). Therefore, statements about activity at one site can only be interpreted with respect to activity at the corresponding site. For simplicity, in the following it will not always be written (e.g., "left-sided activity compared to corresponding right-sided activity," but rather "relative left-sided activity"). In general, just the electrode site of main interest will be mentioned, and it should be noted that activity patterns of different electrode sites in respect to each other are meant.
}

needed to decide whether to accept or reject the gamble. The experiment had two phases, which differed in the contingency between response and acceptance/rejection of the gamble. In Phase 1, participants had to respond for acceptance of the gamble, and in Phase 2, for rejecting the gamble. Participants were paid according to their earnings in the gamble task. The possible wins or losses were presented in form of Experimental Euros (E€), whereby each E€ was worth 2 euro cents (exchange rate 1:50). The experiment started with $50 \mathrm{E} €$. The possible gains ranged from $0-10 \mathrm{E} €$ and possible losses from 0-8 E€, both in steps of $2 \mathrm{E} €$. Possible wins and losses varied over trials in a full factorial combination resulting in 30 different gain-loss combinations. These combinations were allocated into experimental blocks depending on the amount that could be won and lost (see Table 1).

There were trials with a positive net value (win trials), with a negative net value (loss trials), and with comparable amounts of gains and losses (balanced trials). It is important to note that the balanced trials also included positive net values, with gains slightly higher than losses (+2 E€), to take loss aversion into account and to match internal values.

Six experimental blocks were formed: Win trials Phase 1, win trials Phase 2, balanced trials Phase 1, balanced trials Phase 2, loss trials Phase 1, and loss trials Phase 2. Each block was characterized by a distinct motivational tendency. We assumed that win trials Phase 1 and loss trials Phase 2 elicit approach motivation (approach trials), balanced trials conflict (conflict trials), and loss trials Phase 1 and win trials Phase 2 avoidance motivation (avoidance trials). Therefore, a $2 \times 3$ design with phase (Phase 1 vs. Phase 2) and motivational tendency (approach, conflict, avoidance) was derived, as Table 2 shows.

Within each experimental block, the gain-loss combinations were presented in randomized order, and each combination was presented five times, resulting in 50 trials per experimental block and 300 trials in total. All participants were presented the experimental conditions in the same order. Choice behavior, response speed and EEG activity were recorded as dependent variables.

A trial started with the presentation of the particular gain and loss in this trial. Presentation time was 2 seconds, during which the EEG was recorded. Responding was not possible within this timeslot (the computer mouse was disabled and fixed in the middle of the screen), and participants were instructed not to move or try to respond during this period. Then participants had to indicate their decision to play or to abstain (3-second period). A check box randomly appeared at one of four equally distant positions (left top corner, right top corner, left bottom corner, right bottom corner). Participants responded by moving the mouse cursor towards the check box and clicking on it. In Phase 1, a response indicated acceptance of the gamble, whereas no response within 3 seconds indicated rejection of the gamble. In Phase 2, a mouse click on the 
check box indicated rejection of the gamble, whereas no response within 3 seconds indicated acceptance of the gamble. It is important to note that responding had no influence on trial length. Finally, participants received feedback if they had won, lost, or simply kept their money in a no-gamble trial (1.5-second period). The fixed intertrial interval was $2 \mathrm{sec}-$ onds long.

\section{Setting and apparatus}

The experimental room $(4 \times 3.4 \mathrm{~m})$ was sound insulated, air conditioned and had a largely nontechnical appearance. Participants were alone in the experimental room during the experiment. Participants sat comfortably and in an upright position. Experimental control was computerized. Participants were seated in front $(100 \mathrm{~cm}$ distance $)$ of a 22-in. TFTMonitor (VW225N, ASUSTeK COMPUTER INC., Taipei, Taiwan). All experimental information and stimuli were presented on-screen. Participants were instructed to use their right hand for steering the computer mouse (M-5400, Cherry, Auerbach, Germany). EEG, electrooculogram (EOG) and reference electrodes were connected to a Biosemi-Active-2 System (BioSemi, Amsterdam, Netherlands). In the adjoining room, a personal computer $(3.2 \mathrm{GHz}$, Intel Gaming Edition, 4 GB RAM, 1 GB NVIDIA GeForce GT250) that was connected via USB 2 with the Biosemi-Active-2-System performed data recording, data visualization, and storage using ActiView 6.05 (BioSemi, Amsterdam, Netherlands). For experimental control, trigger placement and recording of behavioral responses, a personal computer $(1.6 \mathrm{GHz}, 2 \mathrm{~GB}$ RAM, GB NVIDIA GeForce GT250) was used under MATLAB R2007a (MathWorks, Natick, MA, USA) and the Psychophysics Toolbox 3.0.11 (http://psychtoolbox.org/).

\section{Procedure}

Before the main experiment, participants were asked to fill out an online form that included several personality

Table 1 Combinations of gains and losses in the gamble task

\begin{tabular}{|c|c|c|c|c|c|c|}
\hline & + $0 \mathrm{E} €$ & $+2 \mathrm{E} €$ & + $4 \mathrm{E} €$ & $+6 \mathrm{E€}$ & + $8 \mathrm{E€}$ & + $10 \mathrm{E} €$ \\
\hline - 0 E€ & $\begin{aligned} & 0-0 \\
= & 0 \mathrm{E} €\end{aligned}$ & $\begin{aligned} & 2-0 \\
= & 2 \mathrm{E} €\end{aligned}$ & $\begin{aligned} & 4-0 \\
= & 4 \mathrm{E} €\end{aligned}$ & $\begin{aligned} & 6-0 \\
= & 6 \mathrm{E} €\end{aligned}$ & $\begin{aligned} & 8-0 \\
= & 8 \mathrm{E} €\end{aligned}$ & $\begin{aligned} & 10-0 \\
= & 10 \mathrm{E} €\end{aligned}$ \\
\hline - $2 \mathrm{E} €$ & $\begin{aligned} & 0-2 \\
= & -2 \mathrm{E} €\end{aligned}$ & $\begin{aligned} & 2-2 \\
= & 0 \mathrm{E} €\end{aligned}$ & $\begin{aligned} & 4-2 \\
= & 2 \mathrm{E} €\end{aligned}$ & $\begin{aligned} & 6-2 \\
= & 4 \mathrm{E} €\end{aligned}$ & $\begin{aligned} & 8-2 \\
= & 6 \mathrm{E} €\end{aligned}$ & $\begin{array}{l}10-2 \\
=8 \mathrm{E} €\end{array}$ \\
\hline - $4 \mathrm{E€}$ & $\begin{aligned} & 0-4 \\
= & -4 \mathrm{E} €\end{aligned}$ & $\begin{aligned} & 2-4 \\
= & -2 \mathrm{E} €\end{aligned}$ & $\begin{aligned} & 4-4 \\
= & 0 \mathrm{E} €\end{aligned}$ & $\begin{aligned} & 6-4 \\
= & 2 \mathrm{E} €\end{aligned}$ & $\begin{aligned} & 8-4 \\
= & 4 \mathrm{E} €\end{aligned}$ & $\begin{array}{l}10-4 \\
=6 \mathrm{E} €\end{array}$ \\
\hline - $6 \mathrm{E€}$ & $\begin{aligned} & 0-6 \\
= & -6 \mathrm{E} €\end{aligned}$ & $\begin{aligned} & 2-6 \\
= & -4 \mathrm{E} €\end{aligned}$ & $\begin{aligned} & 4-6 \\
= & -2 \mathrm{E} €\end{aligned}$ & $\begin{aligned} & 6-6 \\
= & 0 \mathrm{E} €\end{aligned}$ & $\begin{aligned} & 8-6 \\
= & 2 \mathrm{E} €\end{aligned}$ & $\begin{array}{l}10-6 \\
=4 \mathrm{E} €\end{array}$ \\
\hline - $8 \mathrm{E} €$ & $\begin{aligned} & 0-8 \\
= & -8 \mathrm{E} €\end{aligned}$ & $\begin{aligned} & 2-8 \\
= & -6 \mathrm{E} €\end{aligned}$ & $\begin{aligned} & 4-8 \\
= & -4 \mathrm{E} €\end{aligned}$ & $\begin{aligned} & 6-8 \\
= & -2 \mathrm{E} €\end{aligned}$ & $\begin{aligned} & 8-8 \\
= & 0 \mathrm{E} €\end{aligned}$ & $\begin{array}{l}10-8 \\
=2 \mathrm{E} €\end{array}$ \\
\hline
\end{tabular}

Note. The columns represent possible gains $(+\mathrm{E} €)$ and the rows represent possible losses $(-\mathrm{E} €)$ during the gamble. There was a full factorial combination of all possible gains and losses. The cells show particular gain-loss combinations and the resulting net value (gain-loss). Net values of gain-loss combinations were used to group the trials into experimental blocks. Green color indicates that trials were grouped into win trials; red color, loss trials; and light-gray color, balanced trials. Here it is important to note that balanced trials not only included net values of zero but also small positive net values $(+2 \mathrm{E} €)$ to take loss aversion into account. The possible wins on losses were presented in form of Experimental Euros (E€), whereby each E€ was worth two euro cents (exchange rate 1:50). 
Table 2 Experimental design

Motivational tendency

\begin{tabular}{lllll} 
& & Approach & Conflict & Avoidance \\
\hline Phase & $\mathbf{1}$ & Win trials $^{1}$ & Balanced trials & Loss trials $^{3}$ \\
& $\mathbf{2}$ & Loss trials $^{6}$ & Balanced trials $^{5}$ & Win trials \\
\hline
\end{tabular}

Note. In Phase 1, participants had to respond for accepting the gamble, and in Phase 2, for rejecting the gamble. Due to this contingency change, different net values should elicit predominantly approach and avoidance behaviors in Phase 1 and Phase 2, respectively. For example, win trials should predominantly lead to approach tendency in Phase 1, but to avoidance tendencies in Phase 2. The monetary incentives that are presumed to provoke the intended motivational tendencies are presented within the cells. Superscripted 1-6 represent the order in which the experimental blocks were presented, starting the experiment with win trials in Phase 1 and ending the experiment with loss trials in Phase 2.

questionnaires (described in more detail below) and questions about the inclusion/exclusion criteria; it lasted about $45 \mathrm{mi}-$ nutes. If participants met all inclusion criteria, they were invited for the main laboratory testing session.

The main session was at least 1 day later and took about 2 hours. A male experimenter conducted the experiment; all participants were tested alone. After applying the electrodes for EEG and EOG, the participants were instructed to sit calmly and to prevent eye movements and blinks in order to help prevent artifacts. Before starting the experiment, a training session (60 trials in 10 minutes) was conducted for familiarizing participants with the experimental procedure. Then, the main experiment was conducted. The experiment was divided in six experimental blocks of about 7 minutes, each representing one experimental condition. Before each block, there was an instruction on the screen to inform the participant which phase of the experiment it was and how to accept/reject the gamble. A pause of 1 minute followed each block, before the next block started. After the third block a pause of 3 minutes was given. In total, the experiment took about 1 hour.

The session ended with a debriefing with questions about the gamble and the motivation that was elicited. Two of these questions were of special interest and were used to assess the motivation provoked by the gambling task ("How much did you desire to win something in the gambles?", "How much did you fear to lose something in the gambles?"). These questions were answered on a 4-point Likert scale $(1=$ not at all, 2 $=$ a little, $3=$ considerable, $4=$ very much $)$.

\section{Personality questionnaires}

As it is still an open debate which personality questionnaires are best suited to capture BAS, FFFS, and BIS sensitivity on the self-report level (Corr, 2016), several personality scales were administered. German versions of the BIS/BAS Scale (Carver \& White, 1994); the Marburg Agentic Extraversion Scale
(MAE; Wacker, 2005); the Reinforcement Sensitivity Theory Personality Questionnaire ( RST-PQ; Corr \& Cooper, 2016); and the scales Social Potency, Achievement, Stress Reactivity and Harm Avoidance of the Multidimensional Personality Questionnaire (MPQ; Tellegen \& Waller, 2008) were used.

To reveal the factor structure of these psychometric scales, an independent study was conducted with $N=290$ students ( $82 \%$ females) that filled out the questionnaires. As it has been argued that especially trait BAS could be a multidimensional construct with different facets (Carver \& Scheier, 1998) and for concrete behavior predictions, specific facets might be more important than the domain traits (Depue \& Collins, 1999), also the subscales of the BAS-inventories were investigated. The data was analyzed with iterated principal factor analysis followed by promax rotation (for previous implementations of the same factor analytical approach, see Comtesse \& Stemmler, 2017; Wacker, Mueller, Hennig, \& Stemmler, 2012).

A six-factor solution (see Table 3) for the principal factor analysis was derived by the scree test (Cattell, 1966), followed by examination of the simple structure (Thurstone, 1947). The factors were interpreted as four correlated BAS factors (BASImpulsivity, BAS-Achievement, BAS-Social Dominance, BAS-Reward Reactivity), one BIS and one FFFS factor.

From a theoretical point of view, the BAS factors BASImpulsivity, BAS-Achievement, and BAS-Reward Reactivity seemed more relevant than BAS-Social Dominance in relation to the current study and the used gambling task. Therefore, we focused further analysis on those three factors.

Since multiple scales indicated similar underlying personality factors, we used factor scores (based on the factor loadings of the prestudy) instead of single questionnaire scales to assess individual scores of BAS-Impulsivity, BASAchievement, BAS-Reward Reactivity, FFFS, and BIS on self-report level. This had the advantage of potentially more reliable estimates of these personality traits and also prevented alpha error inflation when correlating personality traits with behavioral and neuronal variables.

\section{Behavior data analysis}

On the behavioral level, response times and choice behavior were examined as dependent variables. Behavior analysis was done for each participant individually. For the assessment of approach/avoidance sensitivity the overall percentage of approach responses (averaged over all experimental conditions) was investigated. Additionally, response times were used as a graded measure of motivation (Hall et al., 2011). As an indicator for motivation, response times should be influenced by the occurrence of gains and losses. For analyzing relationships between response strength and external incentives, the matching-law can be used (de Villiers \& Herrnstein, 1976; Farley, 1980). Hall et al. (2011) used general equations from 
Table 3 Iterated principal factor analysis of facets from the trait BAS, BIS, and FFFS spectrum (promax rotation)

\begin{tabular}{|c|c|c|c|c|c|c|}
\hline \multirow[b]{2}{*}{ Scales } & \multicolumn{6}{|l|}{ Factor } \\
\hline & $1 \mathrm{Imp}$ & 2 BIS & 3 Achiev & 4 Social & 5 Reward & 6 FFFS \\
\hline BIS/BAS-Fun Seeking & .78 & -.05 & -.16 & -.12 & .10 & -.06 \\
\hline RST-PQ-Impulsivity & .73 & .17 & -.15 & .23 & -.10 & -.03 \\
\hline RST-PQ-Reward Interest & .61 & -.11 & .30 & -.07 & .12 & .05 \\
\hline MPQ-Stress Reactivity & .10 & .87 & -.02 & .04 & -.08 & .15 \\
\hline RST-PQ-BIS & .02 & .86 & .00 & -.09 & .08 & -03 \\
\hline MAE-Happiness & .16 & -.63 & -.01 & -.02 & .35 & .17 \\
\hline BIS/BAS-BIS & -.12 & .56 & -.02 & -.08 & .26 & .16 \\
\hline MAE-Achievement & -.09 & -.12 & .89 & -.05 & -.06 & -.02 \\
\hline MPQ-Achievement & -.03 & .11 & .78 & .02 & -.01 & -.11 \\
\hline RST-PQ-Goal-Drive Persistence & -.04 & .00 & .71 & .07 & .09 & .14 \\
\hline MAE-Dominance & -.03 & -.09 & -.02 & .88 & .02 & .04 \\
\hline MPQ-Social Potency & -.01 & -.01 & .04 & .86 & .07 & -.04 \\
\hline RST-PQ-Reward Reactivity & .05 & -.05 & -.05 & .10 & .78 & .00 \\
\hline BIS/BAS-Reward Responsiveness & -.02 & .17 & .06 & .00 & .78 & -.12 \\
\hline MPQ-Harm Avoidance & -.14 & .02 & -.02 & -.02 & -.10 & .58 \\
\hline RST-PQ-FFFS & .04 & .34 & .00 & .01 & .01 & .57 \\
\hline BIS/BAS-Drive & .37 & .13 & .31 & .10 & .00 & -.01 \\
\hline Factor1 Imp & 1 & & & & & \\
\hline Factor 2 BIS & -.28 & 1 & & & & \\
\hline Factor 3 Achiev & .23 & -.01 & 1 & & & \\
\hline Factor 4 Social & .51 & -.26 & .33 & 1 & & \\
\hline Factor 5 Reward & .45 & .01 & .45 & .23 & 1 & \\
\hline Factor 6 FFFS & -.19 & .25 & .09 & -.16 & .35 & 1 \\
\hline
\end{tabular}

Note. $N=290$. Coefficients from the pattern matrix are presented. Bold indicates that loadings from the pattern matrix are $<-.50$ or $>.50$ for a given scale. Communalities in the principal factor analysis with six factors ranged from .49 (MPQ-Harm Avoidance) to .80 (MPQ-Social Potency). Imp = BASImpulsivity; Achiev = BAS-Achievement; Social = BAS-Social Dominance; Reward = BAS-Reward Reactivity. The lower part of the table displays the correlations between the factors

the matching law and adapted them to the analysis of response time data depending on attraction and repulsion values. Moreover, they included parameters of individual approach and avoidance sensitivity in this equation to derive these sensitivities from the analysis of response times (for a detailed explanation, see Hall et al., 2011):

Response speed $=K\left(A\left(\frac{a)-R(r))}{\left(A(a)+R(r)+r_{0}\right)}\right.\right.$

where $K$ is the nominal maximum speed, $a$ is the total attraction value ( $a=a_{\text {intrinsic }}$ [intrinsic gain from responding, e.g., diminishing boredom] $+a_{\text {extrinsic }}$ [monetary attraction value: gain and loss omission]) and $r$ is the total repulsion value ( $r=r_{\text {intrinsic }}$ [intrinsic cost of responding, e.g., effort to click] $+r_{\text {extrinsic }}[$ monetary repulsion value: loss and gain omission]), $A$ is the individual approach sensitivity and $R$ is the individual avoidance sensitivity, $r_{0}$ is the reinforcement of other behaviors. Recorded response time data was fitted with the presented function to estimate the parameters $K, A, R$, $a_{\text {intrinsic }}, r_{\text {intrinsic }}$, and $r_{0}$. A least square fit was applied to the data, using MATLAB R2010a (MathWorks, Natick, MA, USA). To eliminate gain and loss differences, response times were averaged over both phases, for matched attraction (gains Phase $1 \&$ loss Phase 2) and repulsion values (losses Phase $1 \&$ gains Phase 2). As there were gains of $10 \mathrm{E} €$ but no equivalent losses of $10 \mathrm{E} €$, such trials were excluded from the fitting procedure. It is important to note that this method produces only a ratio of approach against avoidance sensitivity.

For the assessment of gain/loss sensitivity, also choice data and response times were surveyed. On the level of binary choices, the overall percentage of accepted gambles was used, as individuals with stronger gain sensitivity should accept more gambles and participants with stronger loss sensitivity should show more risk avoidance behavior and reject more gambles. Additionally, response times were compared between Phase 1 and Phase 2 to assess gain/loss sensitivity. In Phase 1, responses should be motivated by gains, and in Phase 
2 , by the prevention of losses. Response time differences between both phases (with matched attraction and repulsion values) should be explained by different sensitivities for gains and losses. Loss aversion should result in faster response times for Phase 2 compared to Phase 1.

Summing up, for the behavioral assessment of approach/ avoidance and gain/loss sensitivity two different indicator variables were calculated per sensitivity, one based on choice behavior and one based on response times. However, conceptually both kinds of indicator variables should be driven by the same underlying brain-behavioral sensitivities. Therefore, we used principal component analysis (PCA) for data reduction, producing one component indicating behavioral approach/ avoidance sensitivity and one component indicating behavioral gain/loss sensitivity.

\section{EEG recording and analysis}

The EEG was acquired during each trial while the particular gains and losses were presented, but before participants could respond. These segments of EEG recording lasted 2 seconds within each trial and were merged over trials. The EEG was recorded from 32 sites according to the international 10-20 system (Jasper, 1958) using an Active Two (BioSemi, Amsterdam, Netherlands) active electrode system with Driven Right Leg (DRL) and Common Mode Sense (CMS) as active and passive reference, respectively. Although electrodes from 32 sites were recorded, not all electrode sites were analyzed. The analysis focused on the frontal electrodes (AF4/ $\mathrm{AF} 3 ; \mathrm{F} 4 / \mathrm{F} 3 ; \mathrm{F} 8 / \mathrm{F} 7 ; \mathrm{Fz}$ ) that were most frequently investigated in connection with approach and avoidance motivation (Harmon-Jones \& Allen, 1997; Neo \& McNaughton, 2011; Sutton \& Davidson, 1997; Wacker et al., 2008; Wacker et al., 2012). In addition to EEG, flat-type active electrodes (BioSemi, Amsterdam, Netherlands) were applied for gathering vertical and horizontal EOG and off-line EEG references from left and right earlobe. All electrode offsets were kept on a stable level below $25 \mathrm{mV}$. All signals were sampled at $512 \mathrm{~Hz}$.

After acquisition, EEG raw data were converted from 24 bit BDF files into 16 bit CNT files (PolyRex by Jürgen Kayser, http://psychophysiology.cpmc.columbia.edu/ PolyRex.htm) to prepare them for further processing with Brain Vision Analyzer 2.0 (Brain Products, Gilching, Germany). Data were referenced to vertex $(\mathrm{Cz})$ and filtered ( $0.5 \mathrm{~Hz}$ high-pass filter), followed by a visual screening of all EEG, EOG, and reference channels for artifacts. Whenever there was a large artifact, not caused by blinks or eye movements, the entire timeslot for all channels was excluded from further analyses. An independent component analysis (ICA) was conducted, which served to remove artifacts from blinks and horizontal and vertical eye movements. Channels that were very noisy were not included into the ICA, but after ICA back transformation, such excluded channels (maximally two per participant) were estimated by topographic interpolation. Data was then filtered again (Notch filter $50 \mathrm{~Hz}$, low-pass filter $50 \mathrm{~Hz}$ ) and rechecked for artifacts through automatic data inspection and finally a careful visual inspection. The EEG signal was then rereferenced to the linked earlobes (Hagemann, Naumann, \& Thayer, 2001).

Subsequently, data was segmented accordingly to experimental condition and subdivided into 1-second epochs overlapping by $50 \%$. Afterwards, for each artifact-free epoch, a fast Fourier transformation (FFT with 50\% Hamming window) was computed, and the resulting frequency bins were clustered. The low alpha band $(8-10.25 \mathrm{~Hz})$ was analyzed in this study, as in previous studies of frontal EEG asymmetry effects were especially strong in this frequency band (Wacker et al., 2003), and this band was most strongly associated with cortical glucose metabolism (Oakes et al., 2004).

Power values for each band were $\mathrm{ln}$-transformed to normalize distributions for usage in statistical analysis (Davidson, Jackson, \& Larson, 2000). For each experimental condition, within-subjects averages of band power were computed. For each subject, there was a minimum of 75 artifact-free segments per condition ( $M=96.9, S D=4.3)$, for calculating these averages of band power. Then, EEG indices of interest were computed: (1) for frontal EEG asymmetry the difference of lntransformed power at right and left homologous sites was computed [e.g., ASY-F4-F3 = $\ln$ (alpha power@F4) - $\ln$ (alpha power@F3)]; (2) for bilateral frontal activity the sum of lntransformed power at frontal sites was computed [ASY-Fro = $[\ln ($ alpha power@F4) + ln(alpha power@Fz) + $\ln ($ alpha power@F3)]. These asymmetry measures were used for comparing EEG activity between experimental conditions.

For examining individual differences in EEG activity and relating these to behavioral and questionnaire measures, just those asymmetry indices were used that showed a significant effect of experimental conditions on the group level (which were ASY-AF4-AF3, ASY-F4-F3, and ASY-Fro). To get an individual estimation of overall left versus right frontal activity and bilateral frontal activity, we averaged the asymmetry values over all experimental conditions for each subject. These averaged values should reflect a rather tonic neuronal activity, (relatively) independent of the specific motivational condition. However, it has been argued by Coan and Allen $(2003,2004)$ that specific behavioral responses should be associated with changes in frontal activity rather than tonic activity, which was supported by several empirical studies (Coan, Allen, \& McKnight, 2006; Harmon-Jones \& Sigelman, 2001; Papousek \& Schulter, 2002). Therefore, we hypothesized that especially changes in frontal asymmetry might be related to our behavioral sensitivity measures (i.e., increase in relative left-frontal cortical activity due to approach motivation should be associated with higher behavioral approach sensitivity). However, since we had six experimental conditions, all potentially varying in motivational 
content, it was not a priori clear how to calculate an index for increased relative left-frontal cortical activity caused by increased approach motivation (especially as it was not clear whether to expect higher or lower relative left-frontal activity for the conflict condition in comparison to the avoidance condition). Therefore, we chose an approach to calculate the differences in frontal asymmetry without making exact predictions of the ordering between conditions (especially due to the uncertainty regarding conflict and avoidance condition), which can be mathematically described as the standard deviation over the six experimental condition means. This resulted in a measure, for each participant, of how much frontal asymmetry changed over the six experimental conditions.

\section{Statistical analysis}

For all inferential statistical analysis, STATA 13 (StataCorp LP, Lakeway Drive, Texas, USA) was used. The statistical analysis was conducted in three steps.

First, behavior data was analyzed as explained above. For investigation of loss aversion, several $t$ tests were conducted (one-tailed, alpha level $=.05$ ). To investigate the motivation elicited by each experimental condition, a repeated $2 \times 3$ ANOVA, with phase (Phase 1, Phase 2) and motivational tendency (approach, conflict, or avoidance) as withinsubjects factors, and amount of approach responses as the dependent variable was conducted.

Second, for each EEG asymmetry index, differences between experimental conditions were examined by calculating repeated $2 \times 3$ ANOVAs, with phase (Phase 1, Phase 2) and motivational tendency (approach, conflict, or avoidance) as within-subjects factors, followed by a priori specified contrasts. ${ }^{4}$ Moreover, as influences of experimental conditions on EEG activity might just be present for subjects that experienced the experiment as motivating, we also included the participants' reported motivation as a moderator in the ANOVAs. For all ANOVAs, the degrees of freedom were adjusted with Huynh-Feldt correction (Huynh \& Feldt, 1976), in case that the assumption of sphericity was violated (just corrected values are reported).

In a third step, the relationships between behavioral measures, EEG indices, and personality questionnaire scores were investigated. Multiple variables were measured on questionnaire, behavioral, and neuronal level. To prevent alpha error inflation, we used factor scores for the questionnaire data (as

\footnotetext{
${ }^{4}$ A priori contrasts were inferred from the BBMAA and the bilateral frontal BAS model. For investigating the predictions of the BBMAA, frontal EEG asymmetry (relatively stronger left-sided activity is represented by higher values) was compared between the motivational tendencies, with the predictions: approach > conflict, approach $>$ avoidance and conflict $<$ avoidance. For investigating the predictions of the bilateral frontal BAS model, bilateral frontal activity was compared between the motivational tendencies, with the predictions: approach > conflict and approach $>$ avoidance.
}

described above) instead of single questionnaire scales, resulting in scores for BAS-Achievement, BAS-Impulsivity, BAS-Reward Reactivity, BIS, and FFFS traits. The dimensionality of behavioral and EEG data was also reduced by PCA, resulting in only one component representing each construct of interest, which were behavioral approach/avoidance sensitivity, behavioral gain/loss sensitivity, relative left-frontal activity, bilateral frontal activity, changes in relative leftfrontal activity, and changes in bilateral frontal activity. We investigated the relationship of trait BAS factors, trait BIS and trait FFFS with behavioral approach/avoidance and gain/loss sensitivity; the relationship of trait BAS factors, trait BIS and trait FFFS with relative left-frontal cortical activity and the relationship of trait BAS factors and bilateral frontal activity. Finally, we also investigated the relation of behavioral approach/avoidance sensitivity and changes in relative leftfrontal cortical activity, as well as the relation of behavioral gain/loss sensitivity with bilateral frontal activity changes (as bilateral frontal activity was influenced by valuation processes on the group level). These relationships were investigated by linear regression, with robust estimation of standard errors in case of heteroscedasticity (White, 1980). Strong a priori hypothesis predicted that trait BIS should be related to lower approach versus avoidance sensitivity and also stronger relative right frontal activity, thus these regressions were tested one-tailed. Similarly, trait BAS was assumed to be related to higher approach/avoidance sensitivity and stronger relative left-frontal activity, also leading to one-tailed tests. However, since we tested three different BAS-factors (BASAchievement, BAS-Reward Reactivity, and BASImpulsivity), the regressions were corrected for multiple testing, using the Šidák method (Šidák, 1967), resulting in an alpha-level of $.017\left(\mathrm{p}=\left(1-(1-.05)^{1 / 3}\right)=0.017\right)$. All other regressions were tested two-tailed.

\section{Results}

\section{Behavioral approach/avoidance and gain/loss sensitivity}

The average percentage of approach responses, over all experimental conditions, was $M=48.1 \%(S D=3.7 \%)$. Participants showed approach responses in $84.0 \%(S D=8.2 \%)$ of the trials which were assumed to elicit approach motivation, in $46.4 \%$ ( $S D=6.4 \%$ ) of the conflict trials, and in $13.9 \%$ ( $S D=7.7 \%$ ) of trials which were supposed to elicit avoidance motivation. The repeated $2 \times 3$ ANOVA revealed a significant main effect of motivational tendency, $F(2,49)=923.42, p<.001$, but no significant main effect of phase, $F(1,49)=3.44, p=.07$, and no interaction effect, $F(2,49)=1.62, p=.21$, on approach responses. There were more approach responses in the approach condition than in the conflict, $t(49)=22.98, p<$ .001 , and avoidance condition, $t(49)=42.83, p<.001$; in 


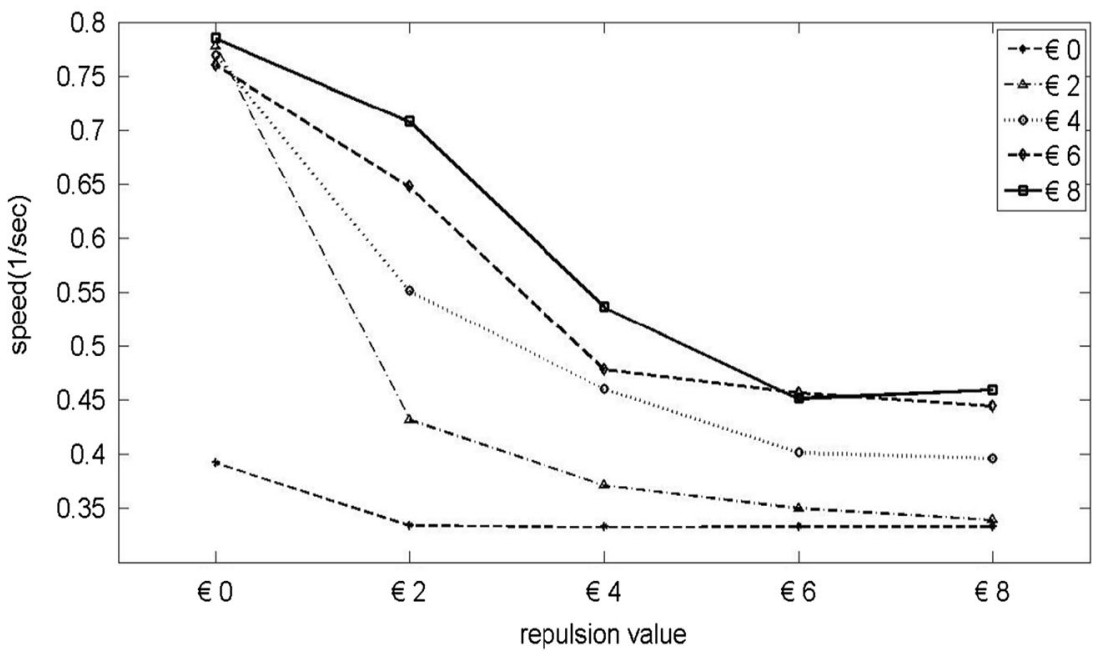

Fig. 1 Observed response speeds $(1 / \mathrm{sec})$ resulting from the combination of a particular attraction and repulsion value, averaged over all participants. Attraction values are averaged over gains and omission of losses (separate values plotted as separate curves). Repulsion values are loss averaged with the omission of gain and is plotted on the $x$-axis. Note the flat-line values at the bottom of the graph are the result of the restriction of a fixed 3 seconds maximum response time addition, the conflict condition showed more approach responses than the avoidance condition, $t(49)=19.85, p<.001$.

Variation in response speed according to changes in attraction value (gain and loss omission) and repulsion value (loss and gain omission) for the averaged data can be seen in Fig. 1. The attraction and repulsion values produced a curvilinear relationship in response speed. Individual response speed data were fitted concurrently to all points with the function derived from the matching law. The fit accounted for $68 \%$ of the variance on average (range: $47 \%-81 \%$ ). For all participants, the individual fitted approach sensitivity was stronger than the avoidance sensitivity, with a mean ratio of approach to avoidance sensitivity of $M=1.99$, but the proportion varied considerably between individuals (range: 1.02-5.20). The percentage of approach responses and the approach/avoidance sensitivity derived from the fitted response time data were significantly correlated $(r=.37, p<.01)$.

In this experiment, $50 \%$ of gambles had higher gains than losses and should be accepted by a rational agent, whereas $16.6 \%$ of trials had equal gains and losses, which should be accepted or rejected equally likely. In total, a rational agent should accept $58.3 \%$ of the trials. The average percentage of accepted gambles was $47.3 \%(S D=10.2 \%)$, which was below the criterion that a rational agent would accept, $t(49)=-7.55$, $p<.001$. Response speed was faster in Phase 2 than Phase 1 , $t(49)=-7.35, p<.001$. Seventy-eight percent of the participants showed faster response times in Phase 2 than in Phase 1, which indicates an inclination for loss aversion. The amount of accepted gambles and the difference in response speed between Phase 1 and Phase 2 were strongly correlated $(r=-.89$, $p<.001)$.

In total, four behavioral variables were assessed for measuring individual approach/avoidance and gain/loss sensitivity. For deriving single variables representing individual approach/avoidance and gain/loss sensitivity, we conducted a PCA over these four behavioral variables. We extracted two components and rotated them with promax rotation. These two components reflected behavioral approach/ avoidance sensitivity (loadings of the variables: approach responses $=.73$; approach/avoidance sensitivity fitted to response time data $=.69$; gambles chosen $=-.02$; response time differences between Phase 1 and Phase $2=-.01$ ) and gain/loss sensitivity (loadings of the variables: approach responses $=$ .06; approach/avoidance sensitivity fitted to response time data $=-.05$; gambles chosen $=.71$; response time differences between Phase 1 and Phase $2=.70$ ), respectively. These two components were not significantly correlated $(r=.17, p>$ $.25)$.

\section{Trait BAS and BIS predict behavioral approach/avoidance sensitivity, but not gain/loss sensitivity}

Behavioral approach/avoidance sensitivity and trait BAS, BIS, FFFS Behavioral approach/avoidance sensitivity was associated with trait BAS-Achievement and trait BIS, as shown in Fig. 2. BAS-Achievement significantly predicted approach/avoidance sensitivity $(\beta=.24), t(49)=2.45, p<$ .01 (Šidák -corrected), with higher trait BAS-Achievement corresponding to higher behavioral approach sensitivity. Moreover, trait BIS significantly predicted approach/ avoidance sensitivity $(\beta=-.20), t(49)=-1.83, p<.05$, Higher trait BIS was associated with lower relative approach sensitivity, or, correspondingly, higher behavioral avoidance sensitivity. BAS-Reward Reactivity, BAS-Impulsivity, and 


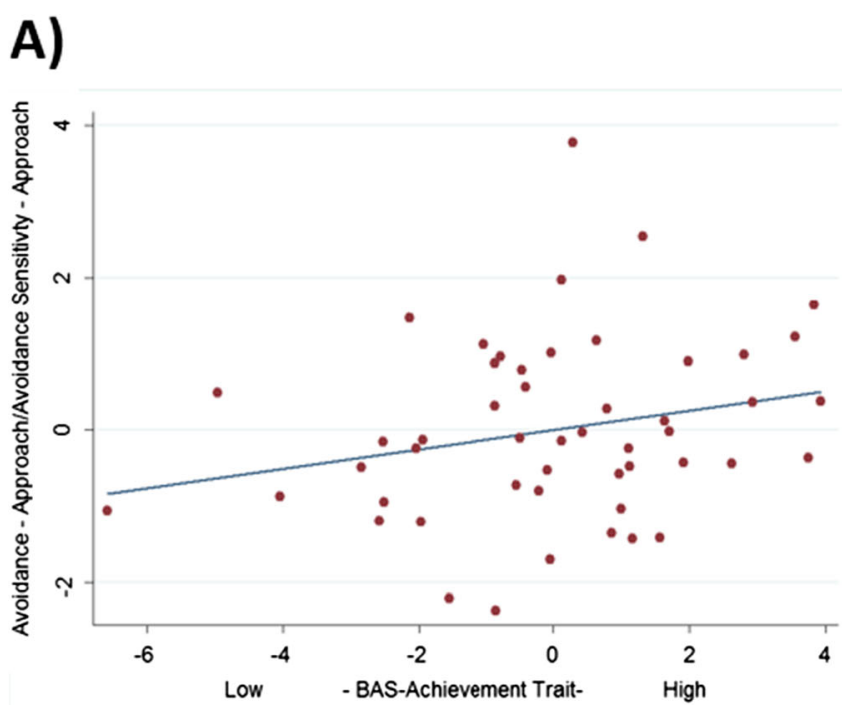

Fig. 2 Scatter plots with regression lines showing the association between behavioral measures of approach/avoidance sensitivity and self-report measures of a trait BAS-Achievement $(\beta=.24 * *)$ and $\mathbf{b}$ trait

FFFS were not associated with behavioral approach/ avoidance sensitivity (all $p \mathrm{~s}>.59$ ).

\section{Behavioral gain/loss sensitivity and trait BAS, BIS, FFFS}

Behavioral gain/loss sensitivity showed no significant associations with trait BAS (all $p \mathrm{~s}>.17$ ), nor with trait BIS and FFFS (all $p \mathrm{~s}>.57$ ).

\section{Frontal EEG asymmetry influenced by motivational tendency}

ASY-AF4-AF3 The ANOVA for ASY-AF4-AF3 showed relatively stronger left-frontal cortical activity in Phase 2 compared to Phase $1, F(1,49)=4.8, p<.05$, and differences in frontal asymmetry between motivational tendencies, $F(2,49)$ $=4.59, p<.05$, but no interaction effect between phase and motivational tendency (see Fig. 3). For differences between the motivational tendencies, a priori contrasts revealed that approach trials showed relatively greater left-lateralized cortical activity than conflict trials, $t(49)=2.51, p<.01$, one-tailed, and avoidance trials, $t(49)=2.63, p<.01$, one-tailed. No difference was found between conflict and avoidance trials.

ASY-F4-F3 The ANOVA for ASY-F4-F3 showed relatively stronger left-frontal cortical activity in Phase 2 compared to Phase $1, F(1,49)=4.41, p<.05$, but no significant effect of motivational tendency and no interaction between phase and motivational tendency. However, there was a significant interaction between motivational tendency and the participants' reported motivation, $F(2,49)=4.84, p<.05$, indicating that the reported motivation moderated the effects of motivational tendency on ASY-F4-F3. Only the subjects that highly feared losing money (answering considerably or very much to the
B)

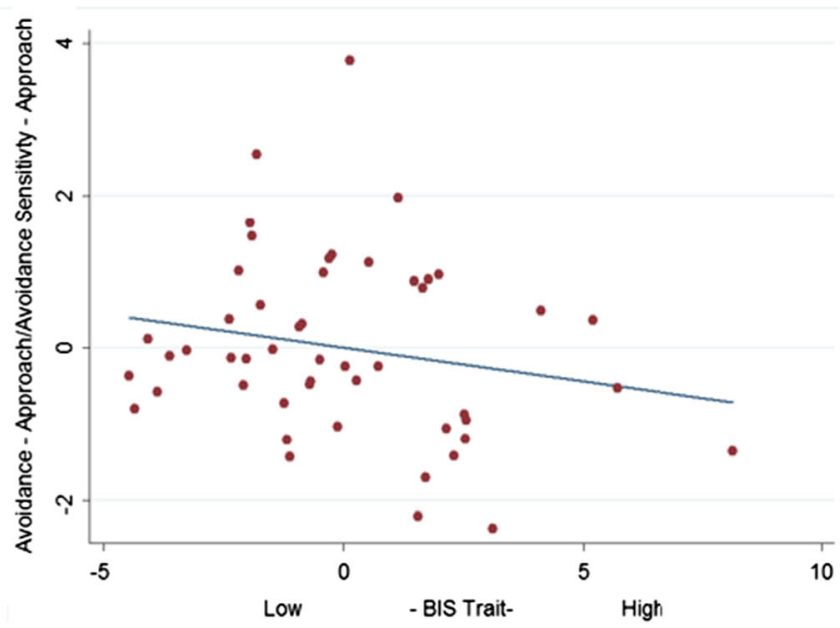

BIS $(B=-.20 *)$. High values for approach/avoidance sensitivity indicate relatively stronger approach sensitivity whereas low values indicate relatively stronger avoidance sensitivity. $* p<.05, * * p<.01$, one-tailed

question of how much they feared losing money) showed a significant effect of Motivational Tendency, with stronger relative left-frontal activity in the approach condition than in the avoidance condition, $t(13)=2.89, p<.01$, one-tailed.

ASY-F8-F7 The ANOVA showed no significant effects, and there was also no moderation present.

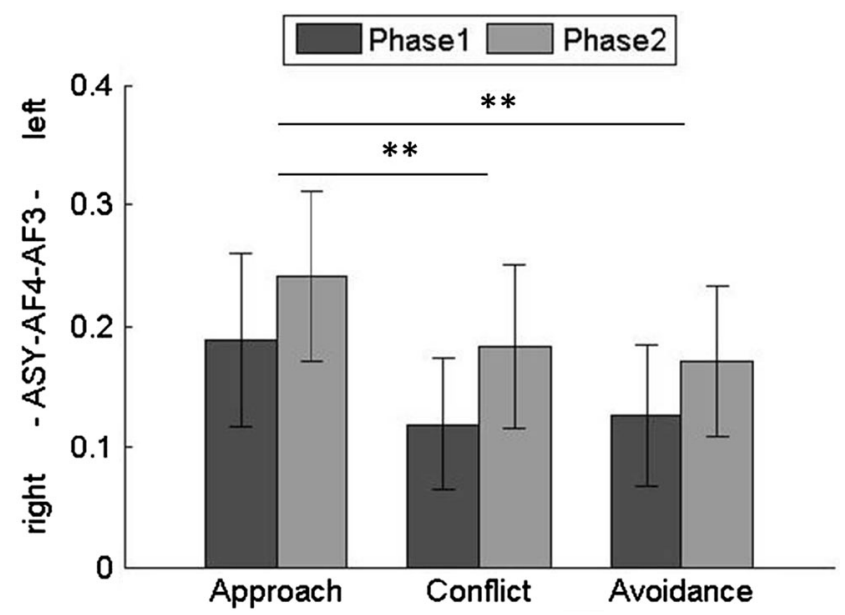

Fig. 3 Differences between right (AF4) and left (AF3) frontal log-power in low alpha band $(8-10.25 \mathrm{~Hz})$ are presented across phase and motivational tendency. Group means and standard errors are presented. Positive values for ASY-AF4-AF3 indicate relative stronger left-frontal activity, whereas negative values would indicate relative stronger right-frontal activity. Significant differences between levels of motivational tendency are marked by asterisks. $* * p<.01$ (unadjusted, one-tailed a priori contrasts between approach-avoidance and approach-conflict). Significant differences between phases are not marked for readability 
Bilateral frontal activity influenced by gain/loss valuation

ASY-Fro The ANOVA for ASY-Fro showed a significant main effect of phase, $F(1,49)=4.38, p<.05$, with stronger bilateral frontal activity in Phase 1 compared to Phase 2, but no main effect of motivational tendency $(p>.22)$. Moreover, there was a significant interaction effect between phase and motivational tendency, $F(2,49)=11.4, p<.001$; see Fig. 4 a. Post hoc simple effect contrasts (two-tailed and Šidák adjusted for three simple effects, between Phase 1 and Phase 2 for all motivational tendencies, and three following contrasts between win, balanced, and loss trials, alpha $=1-(1-.05)^{1 / 6}$ $=.0085)$ revealed that approach trials differed significantly between Phase 1 and Phase 2, $F(1,98)=24.7, p<.001$, with stronger bilateral frontal cortical activity for approach trials in Phase 1 than in Phase 2. Examination of the effects that influenced ASY-Fro revealed that ASY-Fro was affected by the value of the trials (win, balanced, loss) rather than by the motivational tendency. Another $2 \times 3$ ANOVA, with phase and value (win, balanced, loss) as factors, showed a significant main effect of value, $F(2,49)=10.94, p<.001$. Post hoc comparisons revealed stronger bilateral frontal activity for win, $t(49)=4.93, p<.001$, and balanced trials, $t(49)=4.41$, $p<.001$, compared to loss trials (see Fig. 4b). Win and balanced trials did not differ from each other $(p>.43)$.

\section{Indices for individual frontal activity}

In total, three different EEG indices (ASY-AF4-AF3, ASYF4-F3, and ASY-Fro) showed significant influence of experimental conditions on the group level. As we were also interested in individual frontal cortical activity and its relationship with behavioral- and trait-level data, individual scores for overall activity and activity changes due to experimental

A)

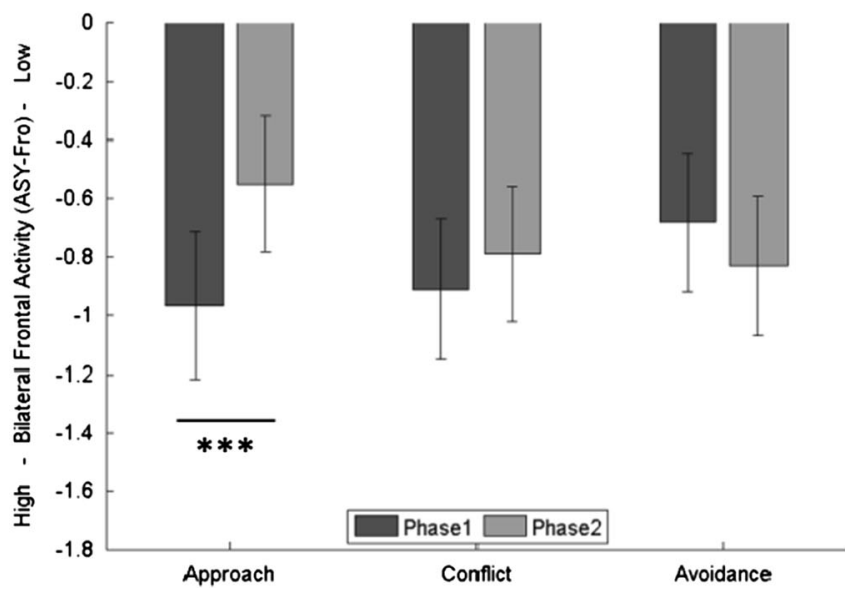

Fig. 4 Bilateral frontal (ASY-Fro $=F 4+F z+F 3) \log$-power $\left(\mu V^{2} / H z\right)$ in low alpha band $(8-10.25 \mathrm{~Hz})$ is presented as group mean and standard error. As the log-power of the alpha band is presented, negative values for ASY-Fro- indicate higher frontal cortical activity, whereas positive values conditions were calculated for each of these indices (as described in the Method section), resulting in a total of six variables for individual frontal cortical activity. We conducted two separate PCAs for dimensionality reduction, one for the overall activity variables and one for variables of activity changes. In both cases, two components were extracted and rotated with promax rotation. The PCA for overall activity resulted in one component representing relative leftfrontal activity (loadings of variables: ASY-AF4-AF3 = .69; ASY-F4-F3 = .73; ASY-Fro $=-.001$ ) and one component representing bilateral frontal activity (loadings of variables: ASY-AF4-AF3 = .09; ASY-F4-F3 = -.09; ASY-Fro = .99). Both components were not significantly correlated $(r=.24, p$ $>$.09). The PCA for activity changes resulted in one component representing changes in relative left-frontal activity (loadings of variables: changes ASY-AF4-AF3 = .68; changes ASY-F4-F3 = .74; changes ASY-Fro = -.001), and the other component represented changes in bilateral frontal activity (loadings of variables: changes ASY-AF4-AF3 = .05; changes ASY-F4-F3 = -.05; changes ASY-Fro = 1.00). Here, both components (changes in relative left-frontal activity and changes in bilateral frontal activity) were significantly correlated $(r=.57, p<.001)$. Interestingly, there was no correlations between overall relative left-frontal activity and changes in relative left-frontal activity as well as no correlation between overall bilateral frontal activity and changes in bilateral frontal activity (all p-values $>.09$ ). Indicating that overall activity and changes in activity represent signatures of different neuronal sensitivities.

\section{Frontal cortical activity correlates with trait BAS}

Relative left-frontal cortical activity was associated with measures of trait BAS (see Fig. 5), whereas no relationship was

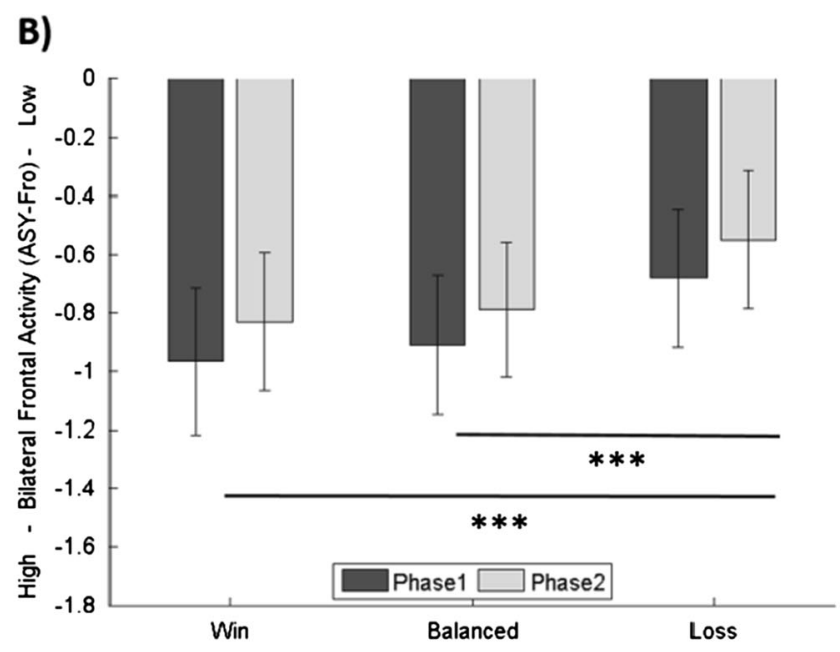

represent lower frontal activity. a Significant simple effects are marked by asterisks. b Significant effects of value (win, balanced, loss) are marked with asterisks. Significant differences between phases are not marked for readability. $* * * p<.001$ 


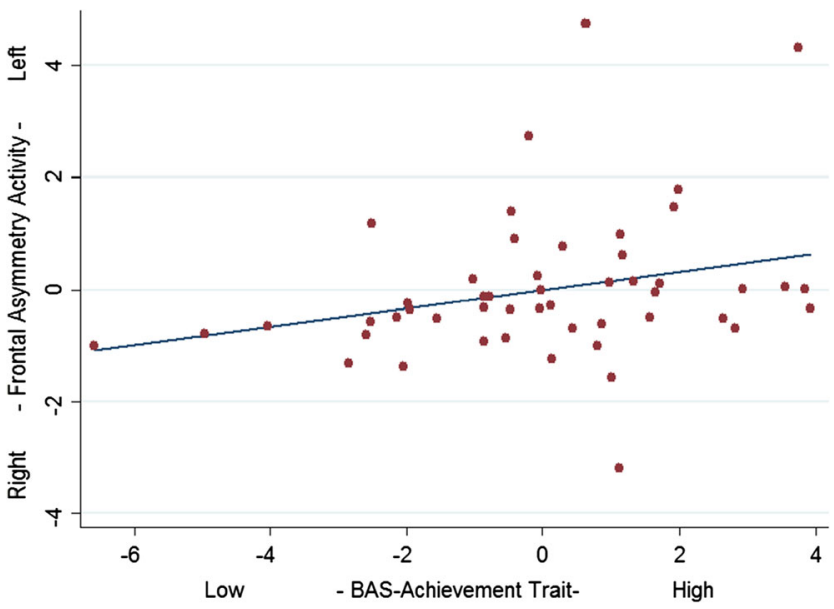

Fig. 5 Scatter plot with regression line showing the association between frontal asymmetry activity and self-report measures of BASAchievement trait $(\beta=.22 *)$. High values for frontal asymmetry activity indicate relatively stronger left-frontal activity, whereas negative values indicate relatively stronger right-frontal activity. ${ }^{*} p<.017$, one-tailed and Šidák -corrected

found between bilateral frontal activity and trait BAS. BASAchievement significantly predicted relative left-frontal cortical activity $(\beta=.22), t(49)=2.21, p<.017$ (Šidák -corrected), with higher BAS-Achievement corresponding to stronger relative left-frontal activity. There was also a significant association between BAS-Reward Reactivity and relative leftfrontal cortical activity $(\beta=.22), t(49)=2.06, p<.05$; however, this relation did not survive the Šidák -correction for multiple comparisons $(p>$.017). Moreover, there was a trend indicating that higher trait BIS corresponded to lower relative left-frontal cortical activity ( $\beta=-.21)$, but this effect did not reach significance $(p=.07)$. BAS-Impulsivity and FFFS were not associated with relative left-frontal cortical activity ( $p$ s > $.15)$.

Additionally, there were no significant relationships between measures of trait BAS and bilateral frontal activity (all ps > .34).

\section{Frontal cortical activity changes predict behavioral approach/avoidance sensitivity}

Changes in relative left-frontal activity predicted behavioral approach/avoidance sensitivity. Gain/loss sensitivity was not associated with bilateral frontal cortical activity changes.

Changes in relative left-frontal cortical activity due to experimental conditions significantly predicted behavioral approach/avoidance sensitivity $(\beta=.50), t(49)=3.56, p<$ .001 . Individuals who showed stronger changes in relative left-frontal cortical activity showed higher behavioral approach sensitivity, as can be seen in Fig. 6 .

Since bilateral frontal activity was influenced by gamble value on the group level, we tested whether bilateral frontal activity would be associated with gain/loss sensitivity on the

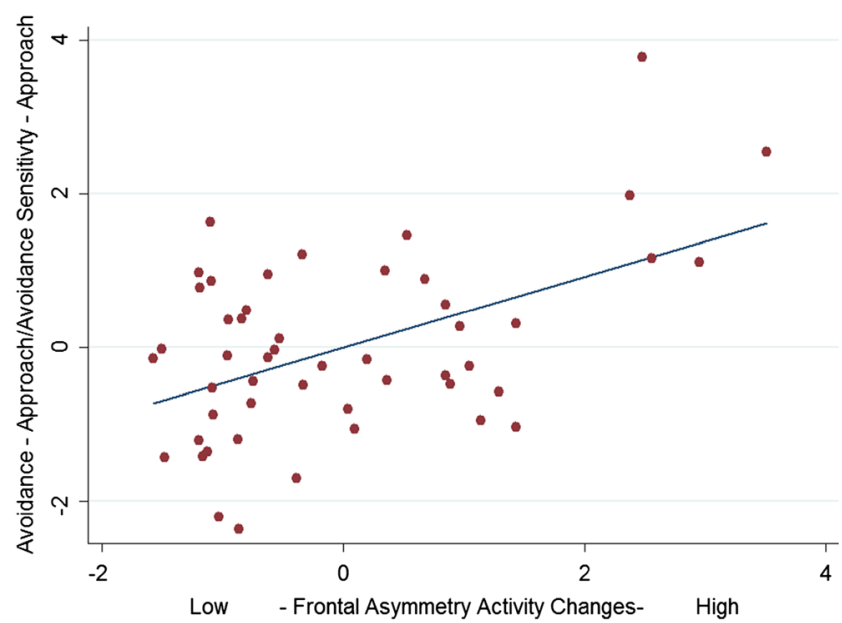

Fig. 6 Scatter plot with regression line showing the association between frontal asymmetry activity changes and behavioral approach/avoidance sensitivity $\left(\beta=.50^{* * *}\right)$. High values in frontal asymmetry activity changes indicate strong changes in relative left-frontal activity due to the experimental conditions, low values indicate small changes due to experimental conditions in relative left-frontal activity. High values for approach/avoidance sensitivity indicate relatively stronger approach sensitivity whereas low values indicate relatively stronger avoidance sensitivity. $* * * p<.001$

individual level. There was no significant association between behavioral gain/loss sensitivity and changes in bilateral frontal activity $(p>.29)$.

\section{Discussion}

This study aimed to assess sensitivities of the brainbehavioral systems proposed by the RST-5, through associated traits and neuronal markers, in order to relate individual differences in these systems to differences in risky decision making. The results showed independent influences of approach/avoidance and gain/loss sensitivities on participants' behavior in risky economic choices. Moreover, individual approach/avoidance sensitivity was predicted by trait measures of BAS and BIS, respectively, while no such correlation was present for gain/loss sensitivity. As predicted by the BBMAA, EEG recordings revealed relatively stronger left-frontal cortical activity for trials with approach motivation compared to conflict and avoidance motivation. In addition, questionnaire measures of trait BAS were associated with stronger relative left-frontal cortical activity. Moreover, activity changes (due to experimental conditions) in frontal asymmetry correlated with behavioral approach/avoidance sensitivity in the sense that stronger neuronal activity changes corresponded to relatively higher behavioral approach sensitivity. In the following, we are going to discuss these main results in more detail. 


\section{Behavioral data}

This study confirmed the behavioral findings of Hall et al. (2011). On the group level, there was an indication for higher loss than gain sensitivity, that is, loss aversion. Analysis of response speed indicated loss aversion due to faster response speed in Phase 2 than in Phase 1. Responses in Phase 2 were motivated by omission of losses, and in Phase 1, by gains. Differences in response speed between both phases should therefore indicate different sensitivities for gains and losses. In accordance, participants accepted less gambles than totally rational agents would have done, which is indicative for stronger loss sensitivity.

The relation between behavioral approach/avoidance sensitivity and gain/loss sensitivity in view of the RST-5, which postulates the distinctness of these processes, is most interesting. We found no relationship between these sensitivities. Accordingly, the distinction between motivational tendency (approach/avoid) and valuation (gain/loss) was supported by the results. This clear distinction might have been accentuated by the experimental design, which was specifically designed to orthogonalize motivational tendency and valuation. In day-to-day life, separation between valuation and motivational tendency could be less pronounced.

Moreover, trait measures of BAS and BIS predicted individual differences in behavioral approach/avoidance sensitivity. This supports the expectation that each response in this paradigm can be seen as approach behavior, mediated by the BAS. Additionally, the data suggest that avoidance behavior is mediated by the BIS rather than the FFFS, because it was a passive form of avoidance. Or staying closer to the assumptions of the RST, the amplification of avoidance motivation by the BIS seemed to be more relevant for this paradigm than the direct control of active avoidance behavior by the FFFS.

But besides the consistent results in our study, it is important to highlight some limitations of these findings and its interpretation. Self-report measures are probably the easiest, but questionably not the best way to assess BAS, BIS, and FFFS sensitivity, as reinforcement sensitivity is probably not well accessible by introspection (Smillie, 2008). A valid test of whether the behavior in this paradigm is actually driven by the BAS and the BIS can only be achieved by a pharmacological manipulation (Corr \& McNaughton, 2012). However, self-report measures should partly represent functional outcomes of neuronal BAS, BIS, and FFFS sensitivities and hence give at least some information about the sensitivities of these brain-behavioral systems (Pickering, 2008).

The absence of a correlation between trait measures and gain/loss sensitivity supports the distinction between valuation and motivation sensitivities and underlines the suggestion that both kinds of sensitivities can vary independently and do not collapse in terms of personality description.

These results show that concepts from the RST-5 prove valuable in predicting individual behavior under risk. However, only valuation sensitivities - uncorrelated with trait measures - were associated with risky behavior in the sense that high gain sensitivity led to riskier behavior (acceptance of more gambles) and high loss sensitivity elicited risk averse behavior (rejecting more gambles). In contrast, motivational sensitivities corresponded to behavioral activity/inhibition, independently of the riskiness of the chosen options. Approach sensitivity represented the tendency to actively reach a decision and strive for attractors by active response behavior, independently of whether the chosen option included a risk (actively accepting the gamble in order to win money) or no risk (actively rejecting the gamble in order to prevent losses). In comparison, avoidance sensitivity represented the tendency to remain passive and accept the default option, also independent of riskiness. Hence, motivational sensitivities were not related to risk proneness/avoidance, per se. Nevertheless, approach and avoidance sensitivity should affect risky behavior indirectly as each risk needs to be actively taken (e.g., deciding to do a base jump) or needs to be accepted passively (e.g., not attending medical checkups), but the relation between motivational sensitivities and risk taking seems rather complex.

\section{Frontal EEG asymmetry is best explained by the BBMAA}

The results showed indications for relatively stronger leftfrontal cortical activity in presence of approach motivation compared with goal conflict or passive avoidance motivation. In addition, individual differences in frontal EEG asymmetry did correlate with trait BAS. Such a pattern of frontal cortical activity can be best explained by the BBMAA. In comparison, the motivational direction model would have also predicted increased left-frontal cortical activity under approach motivation trials, but it would also have assumed stronger rightfrontal activity for avoidance trials compared to conflict trials; however, such a difference was not present.

From an evolutionary perspective, the BIS is believed to protect the organism in situations of potential reward when danger (e.g., a predator) is also present (Corr, 2008). The BBMAA model states that the BIS is most strongly activated by high goal conflict accompanied by relatively right-frontal cortical activity. Differences between conflict and avoidance trials were not found, however. This null finding could speak against the validity of the BBMAA. But goal conflict was probably quite low in the present paradigm and differences between conflict and avoidance trials, hence, quite small, given that each risky choice was followed by gains or losses of only $0.2 €$. According to the BBMAA, a low goal conflict makes it improbable that cortical activity is right-lateralized. 
In addition, the present paradigm probably elicited passive avoidance, which according to the BBMAA also activates the BIS. This makes it even more difficult to find differences between conflict and avoidance trials in frontal cortical activity.

Consistent with the prediction of the BBMAA, relatively stronger left-frontal cortical activity (average over all trials) corresponded with higher trait BAS scores. However, although predicted, there was no significant correlation between relatively right-frontal activity and trait BIS scores. Nevertheless, on a descriptive level, there was a trend between BIS trait and higher relative right-frontal activity. Thus, this null result might be attributed to a slightly underpowered sample size or the rather weak conflict elicited by the task. However, several other studies also did not find an association between right-frontal activity and BIS (e.g. Coan \& Allen, 2000; Harmon-Jones \& Allen, 1997), indicating that the relationship between right-frontal cortical activity and BIS might be quite complex (Coan \& Allen, 2003). Similarly, the expected correlation between trait FFFS and left-frontal activity was not present, probably because of weak involvement/activity of the FFFS. Summing up, this study corroborates other findings that a behavioral approach (or more general, a behavioral activity) system is projecting to left-frontal regions (e.g., Davidson et al., 1990; Sobotka, Davidson, \& Senulis, 1992).

One last interesting note concerning frontal EEG asymmetry was the relatively stronger left-frontal cortical activity in Phase 2 compared to Phase 1 . This cortical activity pattern paralleled the behavioral results of a greater approach motivation in Phase 2, probably elicited by the prevention of losses.

\section{Bilateral frontal cortical activity involved in valuation processes}

The bilateral frontal BAS model predicted increased bilateral frontal activity in the presence of stronger approach motivation, which, however, was not found. Further contradicting this model, individual differences in bilateral frontal activity were not associated with trait BAS.

Interestingly, the results indicate that bilateral frontal activity was involved in processes of valuation rather than motivation: Relatively stronger bilateral frontal cortical activity was present in win and balanced trials compared to loss trials. This finding fits the results of DeMartino, Kumaran, Seymour, and Dolan (2006), who demonstrated that bilateral activity in the vmPFC was associated with the value of a potential choice option. However, in our study, individual differences in behavioral gain/loss sensitivity were not associated with bilateral frontal activity. Whether bilateral frontal activity can explain individual differences in the valuation of gains and losses needs to be shown in future studies, although our results do not indicate such an association.

\section{Changes in frontal EEG asymmetry predict behavioral approach/avoidance sensitivity}

Cortical activity changes predicted behavioral approach/ avoidance sensitivity in this study. Larger increases in relative left-frontal activity corresponded with relatively greater behavioral approach than avoidance sensitivity.

Previous studies have already shown that frontal EEG asymmetry predicts riskiness of behavior (Gianotti et al., 2009) and even showed that disruption of frontal cortical activity by transcranial magnetic stimulation influenced risky behavior (Knoch et al., 2006). But in contrast to our study, Gianotti et al. (2009) did not differentiate between motivation and valuation effects. The authors concluded that frontal EEG asymmetry would predict riskiness of choices, whereas in our study frontal EEG asymmetry predicted the tendency to approach or avoid a situation rather than riskiness itself.

\section{Trait and state nature of frontal EEG asymmetry}

Summing up the results, motivational processes linked to the BAS and BIS were consistently associated with frontal EEG asymmetry. Psychometric BAS traits were linked to left- versus right-frontal cortical activity, but not to activity changes, whereas behavioral approach/avoidance sensitivity, assessed with the gambling task, was associated with activity changes in left- versus right-frontal regions, but not with general activity. Conceivably, there was a distinction between frontal cortical activity and changes in cortical activity that need some consideration.

In this regard, an interesting conceptualization was presented by Coan and Allen (2003, 2004). They differentiated between the state and trait nature of frontal EEG asymmetry (Coan \& Allen, 2003, 2004). The tonic activity in left- and right-frontal cortical areas should be understood as a trait-like measure. Frontal EEG activity should therefore predict the reaction to a broad variety of evocative stimuli and environmental conditions. In contrast, changes in left- versus rightfrontal activity should be understood as state-like measures. Frontal EEG activity changes should therefore mediate emotional and motivational outcomes in response to specific environmental conditions and in particular to emotion stimuli. In the present study we could show that trait and state measures of frontal EEG asymmetry were statistically independent and had distinct relationships to trait BAS and to behavioral approach/avoidance sensitivity.

As personality questionnaires inquire general patterns of behavior and experiences over a broad variety of situations, it seems plausible that scores on personality questionnaires were associated with the overall left- versus right-frontal cortical activity. On the other hand, the measured behavioral approach/avoidance sensitivity was strongly context specific and should be more related to the state than trait nature of 
frontal EEG asymmetry. This expectation was indeed met as behavioral approach/avoidance sensitivity was associated with relative left-frontal activity changes. Although the present study was not intended to investigate the state- and traitlike nature of frontal EEG asymmetry, it lends support to the claim that general frontal EEG activity has a trait-like character and relates to consistent behavior patterns in a broad range of situations, whereas frontal EEG activity changes represent a state-like measure that is associated with motivational changes and responses in specific situations.

\section{Conclusion and outlook}

The two-phase gambling task enabled the independent assessment of behavioral gain/loss and approach/avoidance sensitivity. The results support that valuation and motivation represent two separable processing stages with independent sensitivities, as postulated by the RST-5. Behavioral approach and avoidance sensitivity are linked to the BAS and BIS, respectively, whereas gain and loss sensitivity probably represent behavioral outputs from other brain-behavioral systems.

Moreover, the neuronal evidence suggests that the BAS is projecting in left-frontal areas, as postulated by the BBMAA, whereas the link between BIS and right-frontal activity remains more inconsistent. However, due to spatial resolution limitations of the EEG, other methods like fMRI are needed to draw more elaborated conclusions about the exact location of these brain-behavioral systems. Nonetheless, BAS activation should elicit approach behavior and also correspond to relatively left-frontal cortical activity, whereas BIS activation should initiate behavioral inhibition, passive avoidance and risk assessment, and potentially correspond to relatively right-frontal cortical activity. Moreover, general activity of BAS (and potentially also BIS), corresponding to general activity in left- versus right-frontal cortical regions, could serve as a moderator of motivational responding in a broad variety of situations. In contrast, particular motivated behaviors in specific situations should be mediated by activity changes in left- versus right-frontal cortical regions, in response to motivational and emotional stimuli.

Our study suggests that especially BAS sensitivity can be assessed on the behavioral, neuronal and psychometric trait level, with convergence between the different levels of operationalization. For BIS sensitivity, this convergence was also present between the behavioral and psychometric trait level. Future research should establish the causal link between neuronal and behavioral manifestations of BAS and BIS activity, preferentially with drugs inhibiting or amplifying neuronal signals of these systems (Corr \& McNaughton, 2012). The BAS is tightly linked to the neurotransmitter dopamine (Depue \& Collins, 1999; Pickering \& Smillie, 2008; Schultz, 1998), whereas the BIS should be strongly sensitive to anxiolytic drugs (Blanchard, Griebel, Pobbe \& Blanchard, 2011; Blanchard, 2001; Blanchard,
Griebel, Henrie \& Blanchard, 1997; McNaughton \& Corr, 2008). Therefore, dopaminergic or anxiolytic drugs could be used to more firmly suggest a causal link between behavioral outputs, frontal cortical activity, and BAS/BIS sensitivity. If a causal relationship exists, dopamine agonists should increase relatively left-frontal cortical activity and also behavioral approach sensitivity, whereas anxiolytic drugs should reduce behavioral avoidance sensitivity and potentially also relatively right-frontal cortical activity. Interestingly, two studies showed that an increase in dopamine, due to L-DOPA application, resulted in increases of value-independent gambling (Rigoli, Rutledge, Chew, Ousdal, Dayan \& Dolan, 2016; Rutledge, Skandali, Dayan \& Dolan, 2015) which is very similar to the behavioral approach sensitivity in our task.

In our study, no consistent results concerning neuronal or psychometric correlates of gain and loss sensitivity were found. As most neuronal structures associated with gain and loss valuation are located sub-cortically (amygdala and ventral striatum) or deep inside the skull, like the vmPFC (Trepel \& Poldrack, 2005), other techniques than EEG, such as fMRI, might be more appropriate to assess the neuronal substrate of gain and loss sensitivity (Davidson, 2004).

Stepping back and taking a broader perspective, the study showed that there is considerable inter-individual heterogeneity in regard to choices under risk. The study supports the view that these individual differences can be attributed (at least to some extent) to differences in the valuation of gains and losses as well as in the motivational tendency to approach appetitive or avoid aversive stimuli. Behavioral manifestations of approach and avoidance sensitivity were associated with scores of trait BAS and BIS sensitivity and also with left- versus rightfrontal cortical activity changes. However, behavioral approach and avoidance sensitivity did not directly represent risk taking per se but rather the tendency to show active behavior or to remain passive, respectively, whereas no direct neuronal correlate of risk proneness was found. Most other studies that investigated neuronal correlates of individual risk behavior did not differentiate between motivation and valuation effects (Gianotti et al., 2009). Thus, re-examination of those previous results could change the prevailing conclusions about the neuronal foundation of risky behavior. However, there are also generalization limitations in our study as we just investigated young female students. Since gender (Davidson, 1995), and even the interaction of participant and experimenter gender (Wacker et al., 2008), can have an impact on frontal EEG asymmetry, it remains to be seen whether our results can be replicated in samples with different characteristics. Moreover, risk taking in this study, as in most other studies (e.g. Gianotti et al., 2009; Knoch et al., 2006), was investigated using money as reward and punishment. Although this approach has some great advantages, it remains an open question for future research whether the same results can be obtained with different kinds of incentives and punishments (e.g. food and pain). 
Nevertheless, it seems that the incorporation of traits, as postulated by the RST- 5 , could be a fruitful approach to explain and predict individual behavior in the presence of incentive motivation and to help understand human behavior in an even more fine-grained fashion.

Author notes We are very grateful to Mira-Lynn Chavanon for her great support with EEG issues and to Neil McNaughton for his insightful comments on our paradigm and the general correspondence about the RST. We thank the University of Marburg for the funding of participant reimbursements. All authors declare no competing financial interests.

\section{References}

Blanchard, D. C. (2001). Mouse defensive behaviors: Pharmacological and behavioral assays for anxiety and panic. Neuroscience \& Biobehavioral Reviews, 25(3), 205-218. doi:10.1016/S01497634(01)00009-4

Blanchard, R. J., Griebel, G., Henrie, J. A., \& Blanchard, D. C. (1997). Differentiation of anxiolytic and panicolytic drugs by effects on rat and mouse defense test batteries. Neuroscience and Biobehavioral Reviews, 21(6), 783-789. doi:10.1016/S0149-7634(96)00062-0

Blanchard, D. C., Griebel, G., Pobbe, R., \& Blanchard, R. J. (2011). Risk assessment as an evolved threat detection and analysis process. Neuroscience and Biobehavioral Reviews, 35(4), 991-998. doi:10. 1016/j.neubiorev.2010.10.016

Breiter, H. C., Aharon, I., Kahneman, D., Dale, A., \& Shizgal, P. (2001). Functional imaging of neural resposes to monetary gains and losses. Neuron, 30, 619-639.

Carver, C., \& Scheier, M. (1998). On the self-regulation of rehavior. New York, NY: Cambridge University Press.

Carver, C. S., \& White, T. L. (1994). Behavioral inhibition, behavioral activation, and affective responses to impending reward and punishment: The BIS/BAS scales. Journal of Personality and Social Psychology, 67(2), 319-333. doi:10.1037/0022-3514.67.2.319

Cattell, R. B. (1966). The scree test for the number of factors. Multivariate Behavioral Research, 1(2), 245-276.

Coan, J. A., \& Allen, J. J. B. (2000). Trait frontal alpha asymmetry and its relationship to the behavioral activation system (BAS). In Psychophysiology (Vol. 37, pp. S33-S33). New York, NY: Cambridge University Press.

Coan, J. A., \& Allen, J. J. B. (2003). Frontal EEG asymmetry and the behavioral activation and inhibition systems. Psychophysiology, 40(1), 106-114. doi:10.1111/1469-8986.00011

Coan, J. A., \& Allen, J. J. B. (2004). Frontal EEG asymmetry as a moderator and mediator of emotion. Biological Psychology, 67(1), 7-50.

Coan, J. A., Allen, J. J., \& McKnight, P. E. (2006). A capability model of individual differences in frontal EEG asymmetry. Biological Psychology, 72(2), 198-207.

Comtesse, H., \& Stemmler, G. (2017). Fear and disgust in women: Differentiation of cardiovascular regulation patterns. Biological Psychology, 123, 166-176.

Corr, P. J. (2008). The reinforcement sensitivity theory of personality. Cambridge: Cambridge University Press.

Corr, P. J. (2016). Reinforcement sensitivity theory of personality questionnaires: Structural survey with recommendations. Personality and Individual Differences, 89, 60-64.

Corr, P. J., \& Cooper, A. J. (2016). The reinforcement sensitivity theory of personality questionnaire (RST-PQ): Development and validation. Psychological Assessment, 28(11), 1427-1440.
Corr, P. J., DeYoung, C. G., \& McNaughton, N. (2013). Motivation and personality: A neuropsychological perspective. Social and Personality Psychology Compass, 7(3), 158-175.

Corr, P. J., \& McNaughton, N. (2008). Reinforcement sensitivity theory and personality. In P. J. Corr (Ed.), The reinforcement sensitivity theory of personality (pp. 155-187). Cambridge, UK: Cambridge University Press.

Corr, P. J., \& McNaughton, N. (2012). Neuroscience and approach/ avoidance personality traits: A two stage (valuation-motivation) approach. Neuroscience and Biobehavioral Reviews, 36(10), 2339 2354. doi:10.1016/j.neubiorev.2012.09.013

Davidson, R. J. (1995). Cerebral asymmetry, emotion, and affective style. In K. Davidson \& R. J. Hugdahl (Eds.), Brain asymmetry (pp. 361387). Cambridge, MA: MIT Press.

Davidson, R. J. (2004). What does the prefrontal cortex "do" in affect: Perspectives on frontal EEG asymmetry research. Biological Psychology, 67(1/2), 219-233. doi:10.1016/j.biopsycho.2004.03. 008

Davidson, R. J., Ekman, P., Saron, C. D., Senulis, J. A., \& Friesen, W. V. (1990). Approach-withdrawal and cerebral asymmetry: Emotional expression and brain physiology: I. Journal of Personality and Social Psychology, 58(2), 330.

Davidson, R. J., Jackson, D. C., \& Larson, C. L. (2000). Human electroencephalography. In J. T. Cacioppo \& L. G. Tassinary (Eds.), Handbook of psychophysiology (2nd ed., pp. 27-52). Cambridge: Cambridge University Press.

De Pascalis, V. (2008). Psychophysiological studies. In P. J. Corr (Ed.), The reinforcement sensitivity theory of personality (pp. 261-290). Cambridge: Cambridge University Press.

de Villiers, P. A., \& Herrnstein, R. J. (1976). Toward a law of response strength. Psychological Bulletin, 83(6), 1131.

DeMartino, B., Kumaran, D., Seymour, B., \& Dolan, R. J. (2006). Frames, biases, and rational decision-making in the human brain. Science, 313(5787), 684-687. doi: 10.1126/science.1128356. Frames

Depue, R. A., \& Collins, P. F. (1999). Neurobiology of the structure of personality: Dopamine, facilitation of incentive motivation, and extraversion. Behavioral and Brain Sciences, 22(03), 491-517.

DeYoung, C. G. (2010). Mapping personality traits onto brain systems: BIS, BAS, FFFS and beyond. European Journal of Personality, 24, 404- 407.

Farley, J. (1980). Reinforcement and punishment effects in concurrent schedules: A test of two models. Journal of the Experimental Analysis of Behavior, 33(3), 311-326.

Ferguson, E., Heckman, J. J., \& Corr, P. (2011). Personality and economics: Overview and proposed framework. Personality and Individual Differences, 51(3), 201-209.

Gianotti, L. R. R., Knoch, D., Faber, P. L., Lehmann, D., Pascual-Marqui, R. D., Diezi, C., \& ... Fehr, E. (2009). Tonic activity level in the right prefrontal cortex predicts individuals' risk taking. Psychological Science, 20(1), 33-38.

Glimcher, P. W., \& Rustichini, A. (2004). Neuroeconomics: The consilience of brain and decision. Science, 306(5695), 447-452.

Gonzalez, R., \& Wu, G. (1999). On the shape of the probability weighting function. Cognitive Psychology, 38(1), 129-166.

Hagemann, D., Naumann, E., \& Thayer, J. F. (2001). The quest for the EEG reference revisited: A glance from brain asymmetry research. Psychophysiology, 38(5), 847-857. doi:10.1111/1469-8986. 3850847

Hall, P. J., Chong, C. W., McNaughton, N., \& Corr, P. J. (2011). An economic perspective on the reinforcement sensitivity theory of personality. Personality and Individual Differences, 51(3), 242-247.

Harmon-Jones, E., \& Allen, J. J. B. (1997). Behavioral activation sensitivity and resting frontal EEG asymmetry: Covariation of putative indicators related to risk for mood disorders. Journal of Abnormal Psychology, 106(1), 159. 
Harmon-Jones, E., \& Sigelman, J. (2001). State anger and prefrontal brain activity: Evidence that insult-related relative left-prefrontal activation is associated with experienced anger and aggression. Journal of Personality and Social Psychology, 80(5), 797.

Hewig, J., Hagemann, D., Seifert, J., Naumann, E., \& Bartussek, D. (2004). On the selective relation of frontal cortical asymmetry and anger-out versus anger-control. Journal of Personality and Social Psychology, 87(6), 926.

Hewig, J., Hagemann, D., Seifert, J., Naumann, E., \& Bartussek, D. (2006). The relation of cortical activity and BIS/BAS on the trait level. Biological Psychology, 71(1), 42-53. doi:10.1016/j. biopsycho.2005.01.006

Huynh, H., \& Feldt, L. S. (1976). Estimation of the Box correction for degrees of freedom from sample data in randomized block and splitplot designs. Journal of Educational Statistics, 1(1), 69-82.

Jasper, H. (1958). Report of the committee on methods of clinical examination in electroencephalography. Electroencephalography and Clinical Neurophysiology, 10, 370-375.

Kahneman, D., \& Tversky, A. (1979). Prospect theory: An analysis of decision under risk (pp. 263-291). Econometrica: Journal of the Econometric Society.

Knoch, D., Gianotti, L. R. R., Pascual-Leone, A., Treyer, V., Regard, M., Hohmann, M., \& Brugger, P. (2006). Disruption of right prefrontal cortex by low-frequency repetitive transcranial magnetic stimulation induces risk-taking behavior. The Journal of Neuroscience, 26(24), 6469-6472.

Knutson, B., \& Peterson, R. (2005). Neurally reconstructing expected utility. Games and Economic Behavior, 52(2), 305-315.

Knyazev, G. G. (2007). Motivation, emotion, and their inhibitory control mirrored in brain oscillations. Neuroscience and Biobehavioral Reviews, 31(3), 377-395. doi:10.1016/j.neubiorev.2006.10.004

McNaughton, N., \& Corr, P. J. (2008). The neuropsychology of fear and anxiety: A foundation for reinforcement sensitivity theory. In P. J. Corr (Ed.), The reinforcement sensitivity theory of personality (pp. 44-94). Cambridge, UK: Cambridge University Press.

Monosov, I. E., \& Hikosaka, O. (2012). Regionally distinct processing of rewards and punishments by the primate ventromedial prefrontal cortex. The Journal of Neuroscience, 32(30), 10318-10330.

Neo, P. S.-H., \& McNaughton, N. (2011). Frontal theta power linked to neuroticism and avoidance. Cognitive, Affective \& Behavioral Neuroscience, 11(3), 396-403. doi:10.3758/s13415-011-0038-x

Novemsky, N., \& Kahneman, D. (2005). The boundaries of loss aversion. Journal of Marketing Research, 42(2), 119-128. doi:10.1509/jmkr. 42.2.119.62292

Oakes, T. R., Pizzagalli, D. a., Hendrick, A. M., Horras, K. A., Larson, C. L., Abercrombie, H. C., \& ... Davidson, R. J. (2004). Functional coupling of simultaneous electrical and metabolic activity in the human brain. Human Brain Mapping, 21(4), 257-270. doi:10. 1002/hbm.20004

Papousek, I., \& Schulter, G. (2002). Covariations of EEG asymmetries and emotional states indicate that activity at frontopolar locations is particularly affected by state factors. Psychophysiology, 39(3), 350 360.

Pickering, A. D. (2008). Formal and computational models of reinforcement sensitivity theory. In P. J. Corr (Ed.), The reinforcement sensitivity theory of personality (pp. 453-481). Cambridge: Cambridge University Press.

Plassmann, H., O’Doherty, J. P., \& Rangel, A. (2010). Appetitive and aversive goal values are encoded in the medial orbitofrontal cortex at the time of decision-making. The Journal of Neuroscience: The Official Journal of the Society for Neuroscience, 30(32), 1079910808. doi:10.1523/JNEUROSCL.0788-10.2010
Rigoli, F., Rutledge, R. B., Chew, B., Ousdal, O. T., Dayan, P., \& Dolan, R. J. (2016). Dopamine increases a value-independent gambling propensity. Neuropsychopharmacology, 41(11), 2658-2667.

Rutledge, R. B., Skandali, N., Dayan, P., \& Dolan, R. J. (2015). Dopaminergic modulation of decision making and subjective wellbeing. Journal of Neuroscience, 35(27), 9811-9822.

Schultz, W. (1998). Predictive reward signal of dopamine neurons. Journal of Neurophysiology, 80(1), 1-27.

Shackman, A. J., McMenamin, B. W., Maxwell, J. S., Greischar, L. L., \& Davidson, R. J. (2009). Right dorsolateral prefrontal cortical activity and behavioral inhibition. Psychological Science, 20(12), 1500 1506.

Šidák, Z. (1967). Rectangular confidence regions for the means of multivariate normal distributions. Journal of the American Statistical Association, 62(318), 626-633.

Smillie, L. D. (2008). What is reinforcement sensitivity? Neuroscience paradigms for approach-avoidance process theories of personality. European Journal of Personality, 22(5), 359-384.

Sobotka, S. S., Davidson, R. J., \& Senulis, J. A. (1992). Anterior brain electrical asymmetries in response to reward and punishment. Electroencephalography and Clinical Neurophysiology, 83(4), 236-247.

Sutton, S. K., \& Davidson, R. J. (1997). Prefrontal brain asymmetry: A biological substrate of the behavioral approach and inhibition systems. Psychological Science, 8(3), 204-210.

Tellegen, A., \& Waller, N. G. (2008). Exploring personality through test construction: Development of the Multidimensional Personality Questionnaire. The Sage Handbook of Personality Theory and Assessment, 2, 261-292.

Thurstone, L. L. (1947). Multiple factor analysis. Chicago: University of Chicago Press.

Tom, S. M., Fox, C. R., Trepel, C., \& Poldrack, R. A. (2007). The neural basis of loss aversion in decision-making under risk. Science, 315(5811), 515-518.

Trepel, C., Fox, C. R., \& Poldrack, R. A. (2005). Prospect theory on the brain? Toward a cognitive neuroscience of decision under risk. Cognitive Brain Research, 23(1), 34-50.

Wacker, J. (2005). Dopamin, Handlungssteuerung und Spontan-EEG: Bausteine einer psychobiologischen Extraversionstheorie. Berlin: Logos.

Wacker, J., Chavanon, M.-L., Leue, A., \& Stemmler, G. (2008). Is running away right? The behavioral activation-behavioral inhibition model of anterior asymmetry. Emotion, 8(2), 232.

Wacker, J., Heldmann, M., \& Stemmler, G. (2003). Separating emotion and motivational direction in fear and anger: effects on frontal asymmetry. Emotion, 3(2), 167-193. doi:10.1037/1528-3542.3.2.167

Wacker, J., Mueller, E. M., Hennig, J., \& Stemmler, G. (2012). How to consistently link extraversion and intelligence to the catechol-Omethyltransferase (COMT) gene: On defining and measuring psychological phenotypes in neurogenetic research. Journal of Personality and Social Psychology, 102(2), 427.

White, H. (1980). A heteroskedasticity-consistent covariance matrix estimator and a direct test for heteroskedasticity. Econometrica: Journal of the Econometric Society, 48(4), 817-838.

Yacubian, J., Gläscher, J., Schroeder, K., Sommer, T., Braus, D. F., \& Büchel, C. (2006). Dissociable systems for gain- and loss-related value predictions and errors of prediction in the human brain. The Journal of Neuroscience: The Official Journal of the Society for Neuroscience, 26(37), 9530-9537. doi:10.1523/JNEUROSCI. 2915-06.2006 